\title{
Impact of Radiology Information System on CT Scan Reporting Time
}

\author{
Fahad Almutairi*, Jaber Alyami \\ Department of Diagnostic Radiology, Faculty of Applied Medical Science, King Abdulaziz, University (KAU), Jeddah, \\ Saudi Arabia \\ Email: ^ffalmutairi@kau.edu.sa
}

How to cite this paper: Almutairi, F. and Alyami, J. (2021) Impact of Radiology Information System on CT Scan Reporting Time. Open Journal of Medical Imaging, $11,73-84$.

https://doi.org/10.4236/ojmi.2021.113007

Received: August 15, 2021

Accepted: September 15, 2021

Published: September 18, 2021

Copyright (c) 2021 by author(s) and Scientific Research Publishing Inc. This work is licensed under the Creative Commons Attribution International License (CC BY 4.0).

http://creativecommons.org/licenses/by/4.0/

(c) (i) Open Access

\begin{abstract}
The medical sector values time when it determines life in its totality. Any waste of time, especially in critical conditions, compromises patients and puts lives at stake. From a diagnosis and treatment perspective, efficient use of time determines the success of procedures. Whether it be the inclusion of computing technologies or it be the implementation of informatics, the benefits of medical technology have been tremendous to the healthcare sector. This research has looked at the impact of the Radiology Information System (RIS) on CT reporting time in the King Khalid Hospital (KKH) in the Kingdom of Saudi Arabia. The approach of the study has been quasi-experimental, using the power calculation of a pair of $381 \mathrm{CT}$ scan reports of 40,000 after which the data was collected and analyzed by using SPSS to deduce the impact that RIS has on CT reporting time. The comparison of CT reporting time is done between two distinct timeframes Pre- and Post-installation of RIS. The patients in the current study were organized into three primary categories: emergency patients, inpatients, and outpatients. The results show that the turnaround time was impacted positively with the incorporation of RIS and related technologies in CT scan patients. The outpatient department saw the most improvement among the three categories indicating the highest average percentage of reduction in Turnaround Time. Thus, it was concluded that the RIS has an overall positive impact on CT reporting time.
\end{abstract}

\section{Keywords}

Turnaround Time (TAT), Radiology Information System (RIS), Computed Tomography (CT)

\section{Introduction}

The past three decades have seen significant improvement in the global health 
care sector characterized by progress in information technology. Hruby notes that there are no two ways about it, technological developments have made countless interventions to save lives as they continue to improve the overall state of global healthcare [1]. Just like other departments, in order to keep up with the competition, the radiology department needs to increase its efficiency with the changing healthcare system over time ([2] [3]). How prompt the radiology department is in terms of producing the final reports is one factor that can greatly increase efficiency and can then be used as a measure of the efficiency of the radiology department ([4] [5]). Some of the most successful areas of technology application include the digitalisation of health records, enhanced patient care from automated systems in the health care system, and remote treatment and diagnosis, among other milestones in the health care system ([6]).

Radiology Information System (RIS) is one example of the implementation of technology in the healthcare sector. In terms of planning, organization, direction and even providing control over administrative activities, RIS not only helps in the storage and manipulation of information but also, helps in the retrieval of information at the same time ([7]). Additionally, RIS helps in utilization as well as the provision of radiology related facilities and services ([8]). Whether it be informatics implementation or it be the commuting related activities, the benefits seen in healthcare are tremendous ([9]). The RIS, according to Boochever [10], refers to a network software system that is used in the management of the associated data. It is especially useful in tracking radiology imaging, billing, and order information. Some of the critical functions that the RIS performs to make it indispensable in the health care sector include scheduling of activities, management of patients, tracking of patients, results reporting, and billing ([7]).

While the incorporation of RIS with CT, an imaging modality, offers a benefit from the perspective of reporting time, this research proposal looks at the impacts that the RIS has on CT scan reporting time at King Khalid Hospital (KKH) in Hail specifically. As mentioned, the aspect of time in a medical procedure is very important in the sense that any mismanagement of time can result in detrimental results which may even cost the lives of the patients ([11] [12]). It therefore means that while using the technologies in the medical sector, the turnaround time is crucial for the success of the procedures intended ([13]).

The RIS is associated with playing a critical role in enhancing the efficiency and productivity in radiology, as opined by Reiner and Siegel [14]. Before the introduction of RIS, record keeping in operations using CT was paper-based which was more time consuming than a process that starts from physician order finishing with radiology report as asserted by Kahn Jr et al. [15]. It is, however, worth noting that the RIS has its own drawbacks when it is linked with other systems essentially revolving around technical issues ([10] [16]).

There have been many studies that took the challenge to find out the role of RIS in the healthcare sector when it comes to incrementing efficiency ([8] [17] [18] [19] [20]). Radiology is expected to be stronger, faster, and better than what 
it used to be compared to other healthcare environments ([5]). It is worth noting that the need for the quickest turnaround time has long been a part of the practice of radiology ([18] [21]).

Initially, according to a study carried out by Jackson [18], the emphasis placed on turnaround time triggered certain changes based upon available results. According to a survey in 2013 conducted over 86 hospitals, between the years 2009 and 2012, the reporting times that were most problematic in the care settings saw a drop of $54.5 \%$. The study went on to show that the department of emergency which used to take average time of 2 to 4 hours declined to between 30 minutes to 2 hours, and there was drop in inpatient times from up to 24 hours to just 4 to 8 hours. Likewise, outpatient times declined greatly from 24 hours to 4 to 8 hours. These drastic changes demonstrate the significant impact made by the radiology departments surveyed.

Yet, when looking at the impact that the implementation of RIS has on the healthcare sector in other Middle Eastern countries, this element appears to be all but totally unexplored. Thus, can consider this study to be one of the first studies that investigate the impact that RIS has in the national healthcare setting in a Middle Eastern country. There are several studies, For example, Jackson [18]; Lahiri and Seidmann [20]; Inamura et al., [17], where some of the authors explored the impact that implementation of RIS had on turnaround time but this research is particularly focused at King Khalid Hospital in Hail in the Kingdom of Saudi Arabia makes it one of a kind in this field of study.

The healthcare system in the Kingdom of Saudi Arabia (KSA) is of utmost importance to the government ([22]). Additionally, there are great opportunities for growth in this lucrative sector. Thus, this research focuses on identifying the impact that implementation of RIS has on the CT reporting time. Hence, this research presents the detailed data collection methods along with the analyses methods used. Further, this research will go on to not only present the results obtained, but also to discuss the relationship of these results with those obtained in previous studies.

\section{Material and Methods}

\subsection{Study Design and Sample Size}

This research approach makes use of various methods like conducting the study for sample size collection, usage of quasi-experimental research approach for data collection, and using Statistical Package for the Social Sciences SPSS for analyzing the data. Concerning the population of the research study, the focus was on patients who had a CT examination in $\mathrm{KKH}$ during the first half of 2017 and 2018. By using power calculation for data collection which involved collecting 381 reports from archives before RIS installation and 381 reports after the installation. The reason for using this method is based on the fact that it is helping researchers to determine the smallest sample size suitable for the detection of a given test based on the desired level of significance ([23]). For this reason, this 
research employs both inclusion and exclusion criteria. The inclusion criteria require that the reports need to be from KKH patients who had a CT scan, while the exclusion criteria involved the reports of the patients that incomplete or unclear. Following is the description that describes the methods used from sample size formation to data collection, data analyses, and ethical considerations:

\subsection{Data Collection}

The Province of Hail in the Kingdom of Saudi Arabia contains four hospitals. The hospitals include King Khalid Hospital, King Salman Specialist Hospital, Children's Hospital, and Hail General Hospital ([24]). King Khalid Hospital $(\mathrm{KKH})$ is the hospital where this study was conducted. It first opened its doors to patients in 1980. At the beginning of 2016, RIS was installed in KKH to be utilized across all departments of the hospital.

The data collected in this research study revolves around the time of registration of CT scan patients. The other information to be recorded in the collection sheet is the time of the finished radiological report. The collection of the data stretched for six months before and after the implementation of RIS. During data collection, there was a comparison in CT reporting time between the data of Pre- and Post-RIS installation. This duration established the turnaround time of the application of the CT scan with the incorporation of RIS.

The total number of patients who seek CT scans every year at the $\mathrm{KKH}$ is about 40,000 . The determination of the sample was done using power calculation with a confidence level (long-term success rate of the method employed) of 95\% and a confidence interval representing a range of acceptable values for the parameter of interest of 5 . The sample size to be engaged in the study is 381 reports seeking a CT scan at $\mathrm{KKH}$. The 762 reports formed the sample of reports associated with this study in order to represent the full report on RIS which was an approximately 40,000 .

The data was collected from the CT unit archive in the radiology department from the KKH. The collection of data was done on paper with keen consideration to ethics regarding the patients. The documents were approved and obtained from ethics committees at both Swansea University and the Ministry of Health in Saudi Arabia. Thus, the study received the necessary ethical approval. Before the application of the Analysis of variance (ANOVA), there was the use of some descriptive statistics. For either year, there were 127 outside the hospital patients, 127 inside the hospital patients, and 127 emergency cases.

\subsection{Data Analysis}

The data analysis for this study was completed using a Statistical Package for the Social Sciences (SPSS), an IBM software package that is used for interactive statistical analysis (Field, 2013). The question of whether patients could benefit from RIS led to a collection of two samples of reports. The first sample comprised 381 patients obtained in the first half of 2017, while a second sample also 
consisted of 381 patients in the first half of 2018. Ensuring the adequate sample size is of utmost importance to the research; if the sample size selected is too large then it not only leads to the study becoming more expensive but also cumbersome in terms of the presentation of results [25]. Finally, two-way ANOVA test was used for linear analysis between reporting times and the number of CT examinations performed. The data analysis in this quasi-experimental design helps in the evaluation of longitudinal effects of interventions through intervention modeling keeping the pre-intervention data into account while analyzing the overall data ([26] [27]). The reason for the use of the ANOVA test was because of the three departments determined for the selection of case studies in this research, and the fact that the test is favorable for testing three or more variables as described by Curtis, Comiskey, and Dempsey [28].

\subsection{Ethical Considerations}

The ethical guidelines set by the Ministry of Health in Saudi Arabia and by the ethics committee at Swansea University were both strictly adhered to while conducting this study. Ethics in research refers to the broadly set rules, both written and unwritten governing the expectations and behaviors of research scientists ([29]). They help in setting expectations of behavior for all those conducting research into healthcare practices and procedures Though healthcare ethics are by no means new, and have, though technological advancement that has taken place, changed dramatically, still the basics of medical ethics remain the same ([30]). The ethical principles are autonomy, beneficence, non-malfeasance, and justice which are followed by all those who belong to this healthcare sector ([31]). The importance of research ethics revolves around the following: promoting the aims of the research, supporting values, and ensuring that the public trusts the research among others ([29]). Importantly, ethical considerations are important as they uphold patients' privacy at all times by keeping their information secure ([32]). Privacy, confidentiality, and security are the three ethical concepts that flow together and often are used interchangeably ([30]). Healthcare professionals and researchers are obliged to keep patient information safe and intact ([33]). Ethics in research teach a high level of respect for all the patients that arrive for care ([29]). For ethical considerations in this particular research project, two approvals were sought, first from Swansea University and the second from the Ministry of Health in Saudi Arabia. These institutions ensured that the research study described here adhered to all ethical requirements before approval.

\section{Results}

The results presenting the comparison and the effect that RIS installation had on the three departments, Emergency, Inpatient, and Outpatient, are clearly indicated in Figures 1-3. In all the three departments, a reduction in the average turnaround time is obtained in the second sample that is Post-RIS installation 
which indicates that RIS installation had a positive effect on all the departments under consideration. The results of the two-way ANOVA are presented in Figure 4 . The average turnaround times between 2017 and 2018 are statistically significantly different. Installation of RIS has statistically significantly reduced the scan to release time ( $\mathrm{p}$-value $<0.001<0.05$ ). This is a strong evidence supporting the use of the RIS and shows that all types of patients have benefited from it. Thus, this answers the research question.

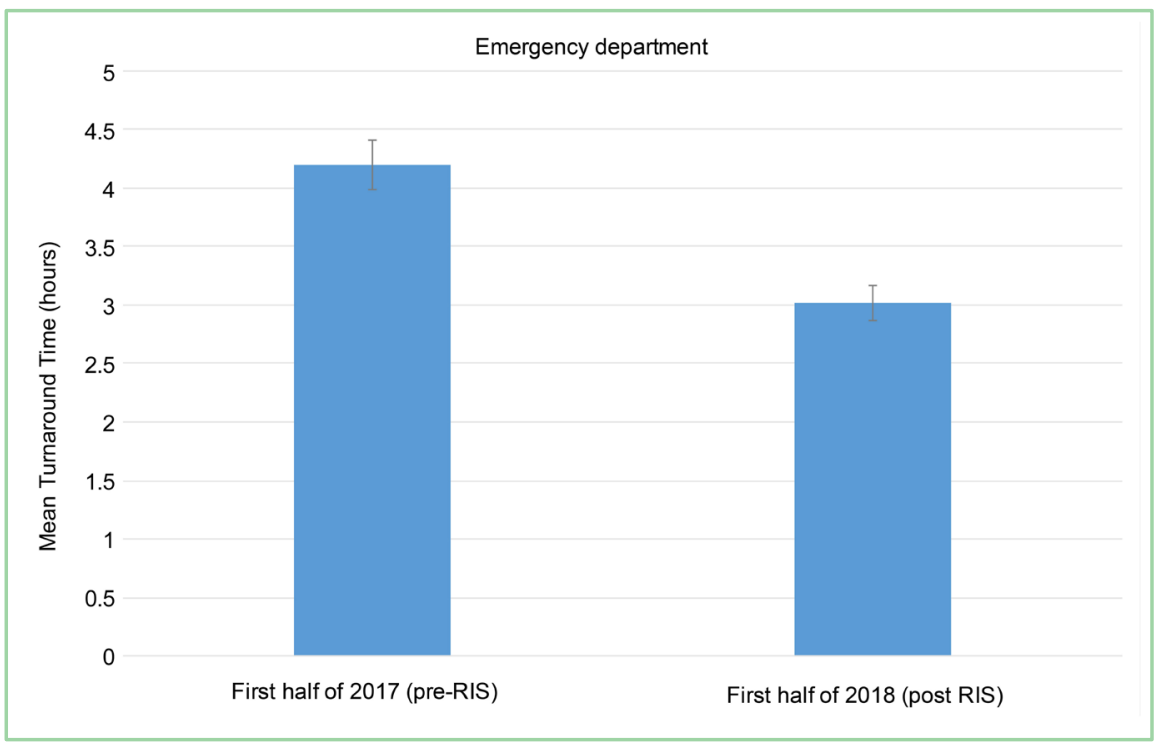

Figure 1. The average turnaround time for Pre- and Post-installation of the RIS for the Emergency Department for the first halves of 2017 and 2018.

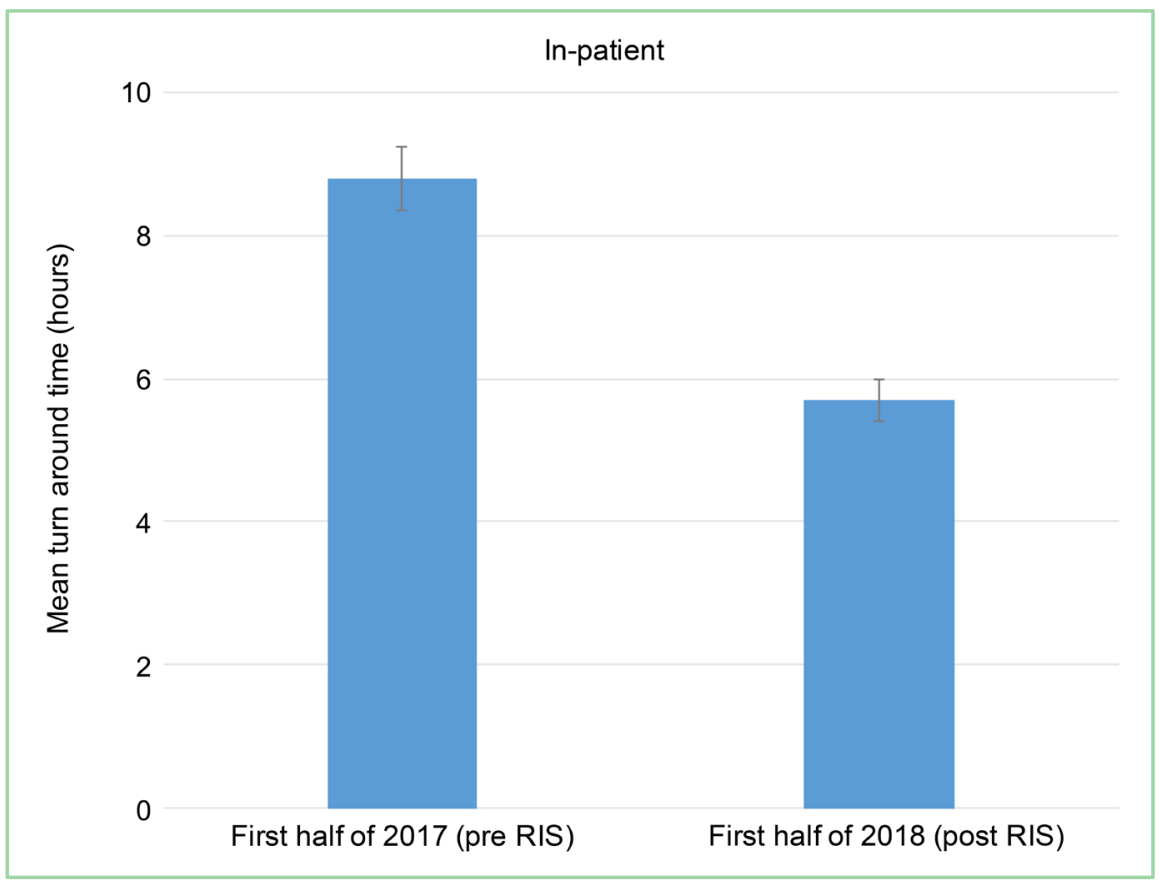

Figure 2. The average turnaround time for Pre- and Post-installation of the RIS for the Inpatient Department for the first halves of 2017 and 2018. 


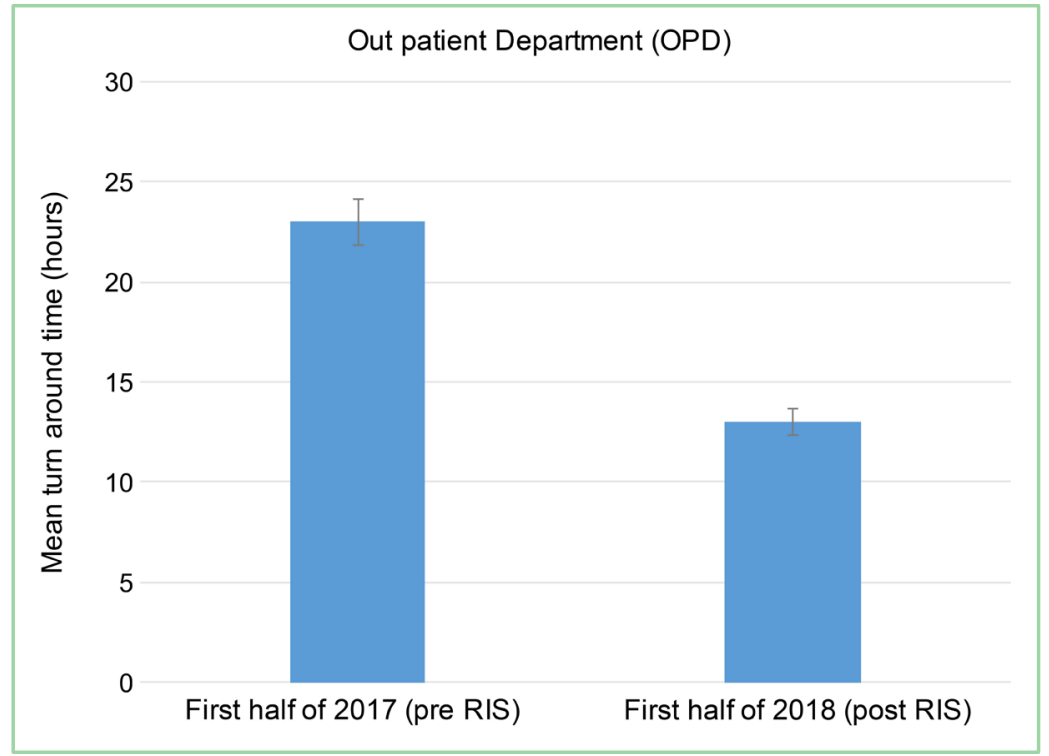

Figure 3. The average turnaround time for Pre- and Post-installation of the RIS for the Outpatient Department for the first halves of 2017 and 2018.

\begin{tabular}{|c|c|c|c|c|c|}
\hline Source & Type III sum of squares & df & Mean Square & $\mathrm{F}$ & Sig. \\
\hline Corrected Model & 38,683 & 5 & 7736 & 1484.73 & $<0.001$ \\
\hline Intercept & 71511.726 & 1 & 71511.726 & 13723.849 & $<0.001$ \\
\hline Department & 30639.455 & 2 & 15319.728 & 2940.016 & $<0.001$ \\
\hline Year & 4770.793 & 1 & 4770.793 & 915.565 & $<0.001$ \\
\hline Department*year & 3263.099 & 2 & 1631.549 & 313.111 & $<0.001$ \\
\hline Error & 3897.651 & 748 & 5211 & & \\
\hline Total & 114198.843 & 754 & & & \\
\hline Corrected Total & 42580.531 & 753 & & & \\
\hline
\end{tabular}

Figure 4. The results of the two-way ANOVA. Results of the two-way ANOVA. Bold are the factors of interest and their associated p-values.

As for their average scan to release time, Figures 1-3, Figure 5, Figure 6 depict this information visually. For the Emergency type of patients, there is a $28.53 \%$ reduction in time, from nearly 4 hours the average time reduced to nearly 3 hours. For the Inpatients, the reduction was more than Emergency Department. From almost 8.5 hours the average scan to release time decreased to roughly 5.5 hours, resulting in a $36.29 \%$ decrease. Outpatients also benefited from the RIS as their average turnaround time was reduced by nearly 11 hours, from almost 24 hours to almost 13 hours (a $45.06 \%$ reduction in time).

The Figure above shows the mean Turnaround Time which used to be higher during the first half of 2017 (Pre-RIS installation) and which shows a decrease after the installation of RIS in the Emergency Department. 


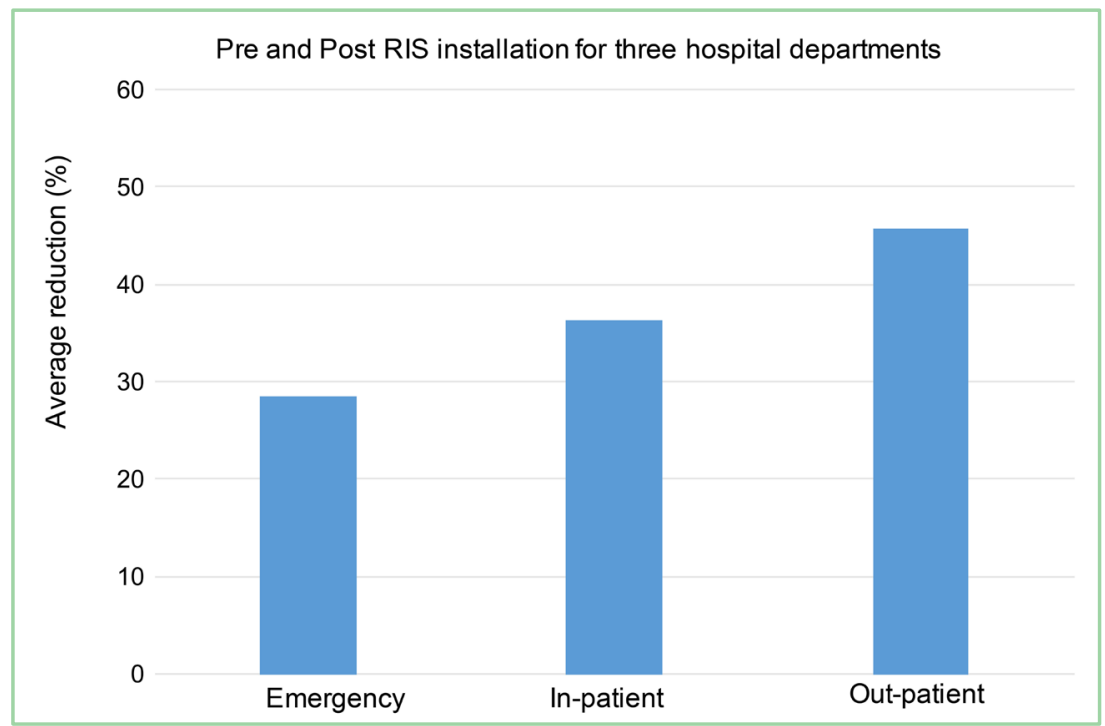

Figure 5. The average percentage reduction in turnaround time between Pre- and Post-installation of the RIS for three hospital departments.

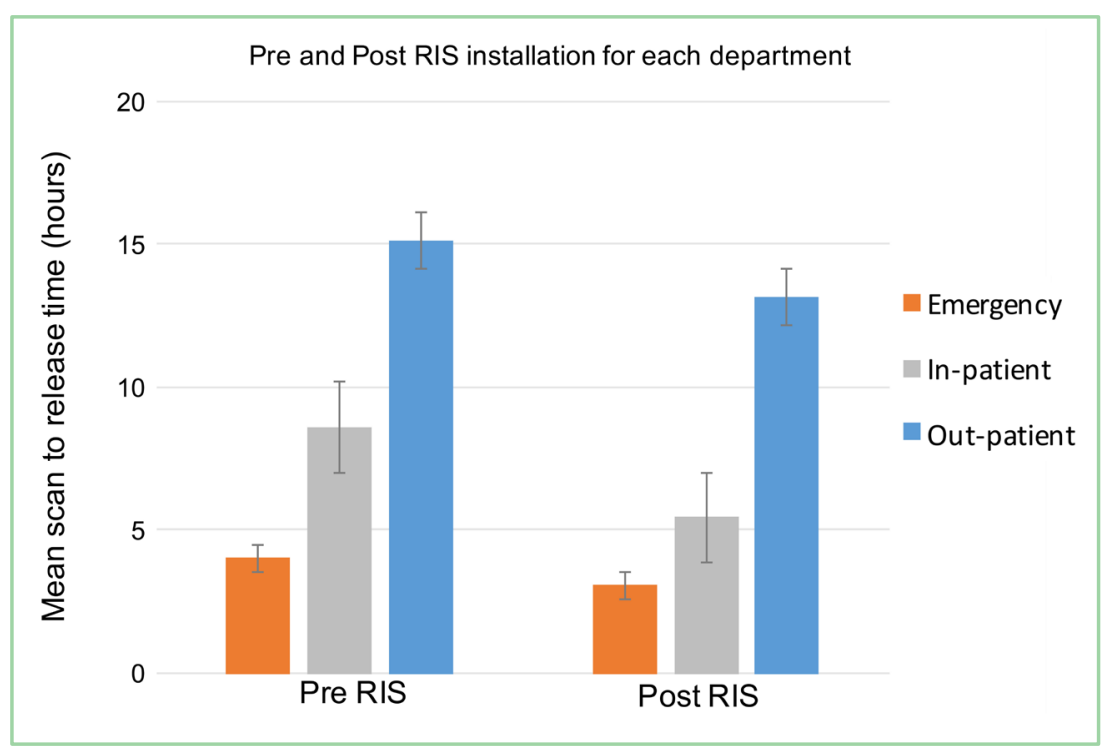

Figure 6. Average scan to release time Pre- and Post- the installation of the RIS for each department.

Similar to the positive effects that RIS installation had on the Emergency Department, Figure 2 and Figure 3 presented above clearly demonstrate that mean Turnaround Time was greatly reduced after the installation of RIS in the Inpatient as well as Outpatient Departments.

These results indicate that the TAT for the Emergency cases provided a reduction of $28.53 \%$, while the reduction in TAT for hospital Inpatients was at $36.29 \%$, and for hospital Outpatients, a reduction of $45.06 \%$.

\section{Discussion}

This research study has looked at the impact of the Radiology Information Sys- 
tem (RIS) on Computed Tomography (CT) reporting time. The general goal of the research study was to establish whether the RIS has an impact on CT reporting time. The search study took place at a health facility called King Khalid Hospital (KKH) in Hail in the Kingdom of Saudi Arabia. The research objective resulted in establishing average turnaround time between periods of research.

From the drastic reduction in Turnaround Time in all the three departments Post-RIS installation, it can be noted that the impact of technology in healthcare systems knows no boundaries and even if boundaries do exist then with the immense list of advantages that technology adds to healthcare, they can be overcome with time.

The results obtained were meant to address the primary research question: is Turnaround Time reduced significantly Post-RIS installation or not? The majority of the results were either tabulated or represented in graphs as indicated in the previous section. As indicated in the graphs above, there was a significant reduction in the release time. As per the results, a drop in the turnaround time (28\% - 45\%) was evident after the installation of RIS, a much lower TAT than what was obtained in other studies (54.5\%) ([18]). It thus represents strong evidence that supports the use of RIS, showing that all the patients surveyed have benefited from the implementation of the system.

Consistency in the results can also be seen when compare the results to other studies which too showed consistency in terms of reduction of turnaround time with the implementation of RIS ([17]). This research study indicates that departments have significant disparity when they are compared on the basis of the time of the scan to its release. In other words, the average scan to the time of release is not similar between departments, as indicated in Figure 5 and Figure 6 . Something to note also is that the scan results of the emergency cases reveal the lowest TAT, followed by the results from the inside of the hospital and, lastly, the hospital outpatients. These results indicate that the turnaround times for the emergency cases had a reduction of $28.53 \%$, while the reduction in inside the hospital patients was at $36.29 \%$, and the outside the hospital patients had a reduction of $45.06 \%$. Based on the results from Figure 4 (Appendix C), the different levels of reduction in time have statistical significance that is different across the three departments. Just like this research, a significant drop in turnaround time was noticed in other study (80\%) ([8]).

Although, our research along with some other studies turned out to be successful in terms of reduction of Turnaround Time with the implementation of RIS but there are other organisations also which did not notice significant difference ([19]) and for some organisation, there was reduction in TAT but they did not find this reduction to be uniform and unsatisfied complained of non-uniformity ([20]).

\section{Conclusions}

Technology plays a significant role in health care and has brought about revolutionary changes in addressing a variety of health care issues. 
In general, the results of the research study have critical implications for establishing the turnaround times of the three different departments. The department with the highest TAT was the Outpatient Department (45\%), followed by the Inpatient Department (36\%), and then the Emergency Department (28\%) had the least change in mean turnaround time. These results show that there is a substantial positive impact of RIS on CT reporting time.

Talking about the implications, several managerial as well as theoretical implications came out from these findings. Firstly, from the perspective of a healthcare provider, in order to make the operational improvements, a competent integration of informatics systems and workflow is necessary. Secondly, from the perspective of the radiology department, there is a need for qualified personnel in the relevant departments who possess the necessary skill set to operate on computers and various pieces of technology just like informaticians. Thirdly, one major implication is related to potential space available post the implementation work is done as this ensures enhanced interface between the other departments of the hospital and the Radiology Department and concerned physicians. Another implication is beneficial to the entire healthcare facility as it can help elevate the level of services by the development of complementary procedures in all the departments.

\section{Acknowledgements}

The authors would like to acknowledge support from Dr. Mubarabk Al Anazi in ethical application and data collection.

\section{Conflicts of Interest}

The authors declare no conflicts of interest regarding the publication of this paper.

\section{References}

[1] Hruby, W. (2013) Digital (R) Evolution in Radiology. Springer Science \& Business Media, Berlin.

[2] Geis, J.R. (2007) Medical Imaging Informatics: How It Improves Radiology Practice Today. Journal of Digital Imaging, 20, 99-104. https://doi.org/10.1007/s10278-007-9010-2

[3] Langlotz, C.P. (2009) Structured Radiology Reporting: Are We There Yet? Radiology, 253, 23-25. https://doi.org/10.1148/radiol.2531091088

[4] Boland, G. (2006) Stakeholder Expectations for Radiologists: Obstacles or Opportunities? Journal of the American College of Radiology, 3, 156-163. https://doi.org/10.1016/j.jacr.2005.10.008

[5] Larson, D.B., Duncan, J.R., Nagy, P.G. and Kruskal, J.B. (2014) Guide to Effective Quality Improvement Reporting in Radiology. Radiology, 271, 561-573. https://doi.org/10.1148/radiol.14131930

[6] Halsted, M.J. and Froehle, C.M. (2008) Design, Implementation, and Assessment of a Radiology Workflow Management System. American Journal of Roentgenology, 191, 321-327. https://doi.org/10.2214/AJR.07.3122 
[7] Babić, R.R., Milošević, Z., Đinđić, B. and Stanković-Babić, G. (2012) Radiology Information System. System (RIS), 1, 11.

[8] Ayal, M. and Seidmann, A. (2009) On the Economic Role of RIS/PACS in Healthcare. SSRN Electronic Journal, 1-9. https://doi.org/10.2139/ssrn.973798

[9] Nance Jr., J.W., Meenan, C. and Nagy, P.G. (2013) The Future of the Radiology Information System. American Journal of Roentgenology, 200, 1064-1070. https://doi.org/10.2214/AJR.12.10326

[10] Boochever, S.S. (2004) HIS/RIS/PACS Integration: Getting to the Gold Standard. Radiology Management, 26, 16-24.

[11] Berlin, L. (1997) Communication of the Significant but Not Urgent Finding. AJR. American Journal of Roentgenology, 168, 329-331. https://doi.org/10.2214/ajr.168.2.9016199

[12] Lu, S.S., Zhang, X., Xu, X.Q., Cao, Y.Z., Zhao, L., Liu, Q.H., Shi, H.B., et al. (2019) Comparison of CT Angiography Collaterals for Predicting Target Perfusion Profile and Clinical Outcome in Patients with Acute Ischemic Stroke. European Radiology, 29, 4922-4929. https://doi.org/10.1007/s00330-019-06027-9

[13] European Society of Radiology (2011) Good Practice for Radiological Reporting. Guidelines from the European Society of Radiology (ESR). Insights into Imaging, 2, 93-96. https://doi.org/10.1007/s13244-011-0066-7

[14] Reiner, B.I., Knight, N. and Siegel, E.L. (2007) Radiology Reporting, Past, Present, and Future: The Radiologist's Perspective. Journal of the American College of Radiology, 4, 313-319. https://doi.org/10.1016/j.jacr.2007.01.015

[15] Kahn Jr., C.E., Langlotz, C.P., Burnside, E.S., Carrino, J.A., Channin, D.S., Hovsepian, D.M. and Rubin, D.L. (2009) Toward Best Practices in Radiology Reporting. Radiology, 252, 852-856. https://doi.org/10.1148/radiol.2523081992

[16] Brady, A.P. (2017) Error and Discrepancy in Radiology: Inevitable or Avoidable? Insights into Imaging, 8, 171-182. https://doi.org/10.1007/s13244-016-0534-1

[17] Inamura, K., Umeda, T., Harauchi, H., Kondoh, H., Hasegawa, T., Kozuka, T. and Inoue, M. (1997) Time and Flow Study Results before and after Installation of a Hospital Information System and Radiology Information System and before Clinical Use of a Picture Archiving and Communication System. Journal of Digital Imaging, 10, 1-9. https://doi.org/10.1007/BF03168543

[18] Jackson, W.L. (2015) In Radiology, Turnaround Time Is King. Practice Management.

https://www.diagnosticimaging.com/pacs-and-informatics/radiology-turnaround-ti $\underline{\text { me-king }}$

[19] Boland, G.W.L., Guimaraes, A.S. and Mueller, P.R. (2008) Radiology Report Turnaround: Expectations and Solutions. European Radiology, 18, 1326-1328.

https://doi.org/10.1007/s00330-008-0905-1

[20] Lahiri, A. and Seidmann, A. (2009) Analyzing the Differential Impact of Radiology Information Systems across Radiology Modalities. Journal of the American College of Radiology, 6, 705-714. https://doi.org/10.1016/j.jacr.2009.05.012

[21] Nitrosi, A., Borasi, G., Nicoli, F., Modigliani, G., Botti, A., Bertolini, M. and Notari, P. (2007) A Filmless Radiology Department in a Full Digital Regional Hospital: Quantitative Evaluation of the Increased Quality and Efficiency. Journal of Digital Imaging, 20, 140. https://doi.org/10.1007/s10278-007-9006-y

[22] Altuwaijri, M. (2011) Health Information Technology Strategic Planning Alignment in Saudi Hospitals: A Historical Perspective. Journal of Health Informatics in De- 
veloping Countries, 5, 18.

[23] Kraemer, H.C. and Blasey, C. (2015) How Many Subjects? Statistical Power Analysis in Research. Sage Publications, London. https://doi.org/10.4135/9781483398761

[24] Ministry of Health (2017) Hail Health Affairs: 537 Medical Devices Delivered to Patients in $1438 \mathrm{H}$.

https://www.moh.gov.sa/en/Ministry/MediaCenter/News/Pages/News-2017-10-08005.aspx

[25] Sathian, B., Sreedharan, J., Baboo, S.N., Sharan, K., Abhilash, E.S. and Rajesh, E. (2010) The Relevance of Sample Size Determination in Medical Research. Nepal Journal of Epidemiology, 1, 4-10. https://doi.org/10.3126/nje.v1i1.4100

[26] Kontopantelis, E., Doran, T., Springate, D.A., Buchan, I. and Reeves, D. (2015) Regression-Based Quasi-Experimental Approach When Randomization Is Not an Option: Interrupted Time Series Analysis. BMJ, 350, h2750.

https://doi.org/10.1136/bmj.h2750

[27] Martin, W.E. and Bridgmon, K.D. (2012) Quantitative and Statistical Research Methods: From Hypothesis to Results (Vol. 42). John Wiley \& Sons, Hoboken.

[28] Curtis, E.A., Comiskey, C. and Dempsey, O. (2016) Importance and Use of Correlational Research. Nurse Researcher, 23, 20-25. https://doi.org/10.7748/nr.2016.e1382

[29] Mallia, P. (2018) Write a Scientific Paper: Ethics Approval for a Research Study (1). Early Human Development, 124, 46-48.

https://doi.org/10.1016/j.earlhumdev.2018.04.022

[30] Rasnik, D. (2015) What Is Ethics in Research \& Why Is It Important? https://www.niehs.nih.gov/research/resources/bioethics/whatis/index.cfm

[31] Sego, S. (2011) Navigating Ethics in a Health-Care Setting-Clinical Advisor. https://www.clinicaladvisor.com/home/features/navigating-ethics-in-a-health-caresetting

[32] Long, T. and Johnson, M. (2007) Research Ethics in the Real World: Issues and Solutions for Health and Social Care Professionals. Elsevier Health Sciences.

[33] Gregory, I. (2003) Ethics in Research. A\&C Black, London. 